Revue d'histoire de l'Amérique française

ZWB REVUE D.HISTOIRE DE L'AMÉRIQUE FRANÇAISE

\title{
Fondation de l'École ménagère de Saint-Pascal (1905-1909)
}

\section{Lucien Lemieux}

Volume 25, numéro 4, mars 1972

URI : https://id.erudit.org/iderudit/303128ar

DOI : https://doi.org/10.7202/303128ar

Aller au sommaire du numéro

Éditeur(s)

Institut d'histoire de l'Amérique française

ISSN

0035-2357 (imprimé)

1492-1383 (numérique)

Découvrir la revue

Citer cette note

Lemieux, L. (1972). Fondation de l'École ménagère de Saint-Pascal (1905-1909).

Revue d'histoire de l'Amérique française, 25(4), 552-557.

https://doi.org/10.7202/303128ar d'utilisation que vous pouvez consulter en ligne.

https://apropos.erudit.org/fr/usagers/politique-dutilisation/ 


\section{NOTE DE RECHERCHE}

\section{FONDATION DE L'ÉCOLE MÉNAGËRE DE SAINT.PASCAL $(1905-1909)^{*}$}

LUCIEN LEMIEUX

L. Th. D. Hist.

\section{Introduction}

En 1897, M. Alphonse Beaudet fut appelé à quitter la cure de Sainte-Philomène pour celle de Saint-Pascal. La situation familiale et sociale, déjà observée dans le comté de Lotbinière, lui paraissait à peu près identique dans celui de Kamouraska. La terre ne produisait pas, les efforts en vue d'enrayer l'émigration vers les villes et vers les Etats-Unis n'aboutissaient à rien ${ }^{1}$, l'ignorance de la femme concernant ses devoirs conjugaux et familiaux était fort répandue ${ }^{2}$. Selon M. Beaudet, les Canadiennes françaises avaient perdu le vrai sens de leurs responsabilités d'épouse, de mère et d'éducatrice ${ }^{3}$. Seule une femme instruite et débrouillarde serait, selon lui, en mesure de ramener le bonheur au foyer. Son époux y restera s'il constate que le produit de son travail se multiplie par l'économie, l'activité et la saine administration de sa femme ${ }^{4}$.

\section{I- Oeurre de collaboration}

1. Accueil du gouvernement provincial

En 1898, le Québec faisait face à une offensive du gouvernement fédéral qui ambitionnait de constituer un organisme de centralisation scolaire. L'honorable Boucher de La Bruère, surintendant de l'Instruction publique, se fit le défenseur acharné de l'autonomie provinciale en matière d'éducation. Par sa bro-

* Ont collaboré à cette recherche les étudiants Anne-Marie Audet, Yvonne-Berthe Bergeron, Jean Patry et Denise-Marie Therrien.

1 ACND (Archives de la Congrégation Notre-Dame), 315.300, 1 (Annales, 1905-1912), discours du 28 juin 1906.

2 ACND, 315.300, 33, Monographie de l'Ecole Normale ClassicoMénagère de la Congrégation Notre-Dame à Saint-Pascal de Kamouraska, 1926. La naissance de l'œuvre.

3 SCNDM (Scurs de la Congrégation de Notre-Dame de Montréal), L'Oeuvre d'un grand éducateur. Le Chanoine Alphonse Beaudet (Montréal, Presses de l'Ecole Industrielle des Sourds-Muets, 1947), I: 34-35.

4 Ibid.

RHAF, vol. 25, no 4 (mars 1972) 
chure de 1904: Education et Constitution, il s'opposa au Central Advisory Sub-Department of Education, projet qui était surtout l'œuvre du Dr J. M. Harper, inspecteur des High Schools du Québec ${ }^{5}$. Il était possible que cette prise de position puisse être accompagnée d'actions concrètes; l'œuvre du curé Beaudet s'insérait dans ce contexte.

Au début de l'année 1904, il rencontra par hasard M. O.-E. Dallaire, directeur de l'Industrie laitière. Celui-ci conseilla au curé de s'adresser au ministère de l'agriculture; il en parlerait lui-même au sous-ministre. Le ministre de l'agriculture, M. A. Turgeon, reçut dès le 10 février une demande d'aide pécuniaire pour une construction dont $M$. Beaudet avait prévu le plan, le coût et le mode de financement. Le Gouvernement se déclara prêt à verser à la future école ménagère une somme annuelle de $\$ 1,500.00$. Une seule condition était posée: avant la mise en opération de l'école, un contrat devait être signé entre la communauté religieuse qui s'en chargerait et le ministère de l'agriculture. De plus, on défraierait le voyage de religieuses que le curé se proposait "d'envoyer en Belgique pour étudier le fonctionnement des écoles ménagères de ce pays" "

2. Démarches fructueuses auprès de la

Congrégation Notre-Dame

M. Beaudet désirait confier la réalisation de son projet à des religieuses canadiennes-françaises, ayant une longue expérience d'éducation au pays. Il s'en ouvrit à sa tante, Sœur Saint-Sulpice, religieuse de la Congrégation Notre-Dame: "Veuillez donc aller aussitôt que possible avec mon ange gardien et celui de notre école ménagère décider vos supérieures à accepter ce que Mère Bourgeoys leur défend de refuser." Le conseil général ne lui laissa pas grand espoir; on lui promit cependant que l'on s'en remettrait à la décision de l'archevêque de Montréal, Mgr Bruchési. Celui-ci prétexta, en présence même du curé, que l'heure n'était pas encore venue d'instaurer une telle forme d'institution au Québec et que la Congrégation Notre-Dame était surchargée. M. Beaudet ne se découragea pas. Il se rendit auprès de Mgr L.-N. Bégin, archevêque de Québec. Celui-ci, qui avait déjà visité et admiré les Ecoles ménagères de Belgique, lui permit de construire l'école; la Providence lui viendrait en aide.

5 Rév. Frère Léopold, Ce secrétariat permanent d'Education. L'histoire d'un demi-siècle de manceuvres centralisatrices. Métamorphoses et camouflages suspects, dans L'Ecole sociale populaire, 353 (juin 1943) : 5-6.

6 SCNDM, op. cit.: 25-26, 29, 31. 
Au début d'août 1904, le projet fut une fois de plus porté devant Mgr Bruchési. Ce dernier répondit: "Cet homme a trop de volonté. Il réussira. Acceptez." Le conseil général accueillit cette décision inespérée "comme un coup du ciel" 7. Mère SaintAnaclet, supérieure générale, communiqua immédiatement à M. Beaudet la réponse de l'archevêque et accepta la direction de l'Ecole pour septembre 1905. La communauté était "heureuse de continuer l'œuvre de sa sainte Fondatrice, la Vénérable Marguerite Bourgeois" 8. Les premières ententes furent signées au presbytère de Saint-Pascal le 20 avril 1905 et le contrat définitif, le 10 août suivant ${ }^{\circ}$. Selon M. Beaudet, la Congrégation, en faisant sienne la lourde dette de plus de $\$ 25,000.00$, avait plus que lui "planté l'arbre" ${ }^{10}$. Les paroissiens célébrèrent la fondation de l'œuvre le 19 août 1905; quatre religieuses furent alors accueillies dans une école à peine terminée ${ }^{11}$.

\section{II - La vie à l'École}

Dès l'automne 1905, les jeunes filles passaient leurs journées entre les classes et les salles de travaux ménagers; cuisine pour peler les légumes et manier la poêle à frire; lavoir pour apprendre à "mousser l'eau" et à "promener le fer"; salles de coupe et de confection où chacune se familiarisait avec l'aiguille et les ciseaux. Le partage des mêmes tâches entre professeurs et élèves créait une ambiance "familiale". On comptait alors huit religieuses et deux laïques, s'occupant à la fois du cours primaire et de l'Ecole ménagère. En 1906, on put répartir les quarante pensionnaires en deux groupes ${ }^{12}$.

A maintes occasions, les jeunes filles exerçaient leur rôle d'hôtesse à l'Ecole même, en apprêtant et servant le repas à des visiteurs: délégués du gouvernement, visiteurs ecclésiastiques ${ }^{13}$. Il leur arrivait aussi d'entrer en contact avec le grand public, soit à l'occasion d'une causerie ${ }^{14}$, soit par un concert ou par une soirée récréative à présenter ${ }^{15}$. La dimension artis-

7 Ibid.: 37 .

8 ACND, 315.300,1, Introduction des Annales.

${ }^{\circ} \mathrm{SCNDM}$, op. cit.: 54 .

10 ACND, 315.200, 1, discours du 28 juin 1906.

11 SCNDM, op. cit.: 51.

12 Ibid.: $92,135$.

13 ACND, 315.300, 1, 13 mars 1909.

14 Ibid., 30 juin 1909.

15 Ibid., 5 mai et 21 novembre 1907 ; 20 avril 1908. 
tique de leur vie se développait en même temps que leurs aptitudes intellectuelles et manuelles ${ }^{16}$. Elles se préparaient à être "servantes" de leur époux et de leurs enfants, mais aussi "reines du foyer", en particulier au salon ou au piano ${ }^{17}$.

L'éducation morale et religieuse s'acquérait comme en symbiose au sein même des multiples activités de l'Ecole. Les $A$ nnales du Couvent mentionnent souvent la participation à la messe, au Salut du Saint-Sacrement, au Mois de Saint-Joseph, au Mois de Marie. Devenir Enfant de Marie faisait l'objet d'une fête particulière. Le curé Beaudet profita aussi de la mission prêchée par les Pères Leclerc et Lamontagne, rédemptoristes, pour offrir aux élèves une retraite spéciale ${ }^{18}$.

Les succès aux examens traduisaient la valeur du travail d'ensemble fait par les élèves. Ces examens se faisaient ordinairement en présence de personnalités marquantes, tels que le surintendant de l'Instruction publique ou son représentant, le ministre de l'agriculture ou le sous-ministre, le curé Beaudet et quelques autres, par exemple le secrétaire provincial ou le député du comté ${ }^{19}$. Ces séances d'examens entretenaient aussi une saine émulation et ne furent pas étrangères au prestige dont bénéficierait l'Ecole de Saint-Pascal. M. Dallaire affirma en 1908 après avoir fait subir un examen aux élèves: "Le système employé à votre Ecole est infiniment supérieur à tous les systèmes d'éducation. C'est l'Ecole qu'on devra avoir partout avant dix ans." 20

\section{III - Affiliation à l'Université}

Dès la fondation de l'Ecole, M. Beaudet avait songé à en assurer le prestige en l'unissant à la plus haute institution d'éducation du pays. Le cardinal Bégin fit part du désir de M. Beaudet à Mgr J.-C.-K. Laflamme, recteur de l'Université Laval de Québec. Les Sœurs elles-mêmes sollicitèrent cette affiliation, le $1^{\text {er }}$ mars 1909 ; elles soumirent alors les grandes lignes de leur programme d'études, éprouvé depuis quatre ans ${ }^{21}$. L'enseignement ménager s'accordait aux trois brevets des autres maisons d'éducation: élémentaire, modèle et académique. Aucun

16 Ibid., 28 juin 1906 et SCNDM, op. cit.: 34.

17 ACND, s15.300, 1, discours du 28 juin 1906.

18 Ibid., 1 mars 1907; 8 décembre 1908.

19 SCNDM, op. cit.: 142.

20 ACND, \$15.800, 1, 28 juin 1906.

21 SCNDM, op. cit.: 125, 25 mai 1909. 
diplôme n'était décerné à des étudiantes de moins de 16 ou 17 ans. Après leur troisième année d'études, elles pouvaient se présenter au certificat d'études ménagères. Après la quatrième année, le diplôme supérieur était possible; il l'était également après la seconde année du cours spécial en économie domestique ${ }^{22}$, lequel fut créé en 1908 pour répondre au désir des élèves qui, ayant terminé leurs études ailleurs, voulaient se former à l'art ménager ${ }^{23}$. En troisième et en quatrième, le programme comportait de l'enseignement classique et ménager ${ }^{24}$.

Une revision attentive du programme d'études de l'Ecole surprit agréablement le comité chargé de l'affiliation; on décida d'aller vérifier sur place en faisant passer des examens aux élèves. Le 28 mai 1909, une délégation se présenta à l'Ecole ménagère de Saint-Pascal: l'honorable Boucher de La Bruère, l'abbé Nadeau, représentant de l'Université Laval, et l'abbé Robert Lagueux. Moins d'un mois plus tard, M. Beaudet recevait un message de sir Lomer Gouin, premier ministre de la province: "J'apprends avec plaisir que votre Ecole Ménagère a été affiliée à l'Université Laval de Québec. Cette nouvelle non seulement me réjouit, mais me fait espérer un avenir de plus en plus brillant pour votre institution." ${ }_{25}$ Il fut en effet confirmé que l'affiliation avait été accordée "par le Conseil Universitaire et par le Conseil de l'Instruction publique réunis à cet effet" ${ }^{26}$.

Quelques jours plus tard, M. Beaudet rappela à la Supérieure générale l'offre gouvernementale d'un voyage en Europe pour des institutrices de l'Ecole. Soeurs S.-M. Vitaline et S.-M. Armand furent choisies pour entreprendre le voyage ${ }^{27}$ et "pour y étudier le fonctionnement des diverses écoles ménagères, celles de Belgique et surtout de Suisse: les plus parfaites du monde, assure-t-on" 28 .

En fait, elles visitèrent les principaux centres d'enseignement ménager d'Europe: l'Ecole ménagère et professionnelle des

22 Ibid.: 147-150.

23 ACND, 315.300, 25, Programme. L'enseignement Classique combiné avec l'Enseignement Ménager (1912), 6.

24 Ibid.: 8-9.

25 SCNDM, op. cit.: 153, 156.

$26 \mathrm{ACND}, 315.300,1,23$ juin 1909, à M. Beaudet.

27 ACND, 315.300, 18 (Visites des principaux centres ménagers européens - 1909), I, 26 et 29 juin, Introduction.

28 ACND, 315.300, 33, Cinq étapes glorieuses. 
Ursulines au Luxembourg, l'Ecole ménagère de Dijon, l'Institut agricole et l'Ecole ménagère de Fribourg ${ }^{29}$. Les deux religieuses rencontrèrent à Paris la comtesse Raoul de Diesback, promotrice de l'enseignement ménager en France et auteur de plusieurs traités en économie domestique ${ }^{30}$. De plus, recommandées par la comtesse d'Oultremont, elles se rendirent à Bruxelles, au ministère de l'industrie, pour s'entretenir avec $M$. Paul Wittouck en l'absence du premier ministre, M. Raubault. Elles y reçurent toutes les informations désirées ainsi que des exemplaires de documents ou de bouquins sur l'organisation de l'enseignement ménager en Belgique ${ }^{31}$. Les deux religieuses rapportèrent aussi d'Europe des cartes de physique, des casiers pour l'étude de la chimie alimentaire, des pièces d'anatomie et d'autres instruments du même genre ${ }^{32}$.

\section{Conclusion}

En même temps qu'une seconde étape, l'affiliation à l'Université Laval fut le tremplin d'une évolution rapide et glorieuse. Quelques mois plus tard, on voulut assurer une valeur pratique aux diplômes universitaires, en accordant à ses titulaires le droit de professer dans toutes les écoles du Québec. Le projet d'Ecole normale fut étudié avec soin. Le 10 mai 1912 , l'archevêque de Québec annonça que la demande avait été agréée par le Comité catholique; le 27 juin 1913, sir François Langelier, lieutenant-gouverneur, approuva l'arrêté ministériel qui conférait à l'Ecole ménagère de Saint-Pascal le titre d'Ecole normale classico-ménagère. En 1915, on ajouterait en conséquence un cours supplémentaire d'une année ${ }^{33}$.

Le sommet fut atteint en 1920: on organisa un cours professionnel ayant pour but de rendre certaines étudiantes aptes à former elles-mêmes des professeurs d'enseignement ménager. Cela requérait une autre année de cours. L'Ecole Saint-Pascal devenait ainsi une Ecole-Mère, qui diffusait de toutes parts lumière et conseil ${ }^{34}$. Elle changerait de nom en 1935 en l'honneur de son fondateur; elle deviendrait l'Institut Alphonse Beaudet ${ }^{35}$.

29 Ibid.

30 ACND, 315.300, 18, I, 30-31, 13 juillet 1909.

31 Ibid., III : 301,28 août.

32 ACND, :15.300, 33, 19 septembre 1909.

33 ACND, 315.300, 33, Cinq étapes glorieuses.

34 Ibid. et SCNDM, op. cit.: 578.

35 SCNDM, (Montréal, Presses de l'Ecole Industrielle des SourdsMuets, 1949) op. cit: II : 379 . 